\title{
Social-Cognitive Contributors to Young Children's Empathic and Prosocial Behavior
}

\section{Citation}

Vaish, Amrisha, and Felix Warneken. 2011. Social-Cognitive Contributors to Young Children's Empathic and Prosocial Behavior. In Empathy: From Bench to Bedside, ed. Jean Decety, 131-146. Cambridge: MIT Press. doi:10.7551/mitpress/9780262016612.003.0008

\section{Published Version}

doi:10.7551/mitpress/9780262016612.003.0008

\section{Permanent link}

http://nrs.harvard.edu/urn-3:HUL.InstRepos:33980469

\section{Terms of Use}

This article was downloaded from Harvard University's DASH repository, and is made available under the terms and conditions applicable to Open Access Policy Articles, as set forth at http:// nrs.harvard.edu/urn-3:HUL.InstRepos:dash.current.terms-of-use\#OAP

\section{Share Your Story}

The Harvard community has made this article openly available.

Please share how this access benefits you. Submit a story.

\section{Accessibility}


Social-Cognitive Contributors to Young Children's Empathic and Prosocial Behavior

Amrisha Vaish and Felix Warneken

To appear in J. Decety (Ed.) (2011), Empathy: From Bench to Bedside. Cambridge: MIT Press. 


\section{Introduction}

Humans, even as young children, act prosocially, that is, in ways that benefit other individuals. Quite early in ontogeny, for instance, children help, comfort, and share resources with others (Hay \& Cook, 2007; Warneken \& Tomasello, 2009). A question that has occupied psychologists for a long time concerns the mechanisms and motivations underlying such prosocial behavior. Investigating this issue is essential to understanding prosociality: First, people might engage in prosocial behaviors for a number of reasons, including selfish ones - to gain approval, be rewarded, or because acting prosocially relieves the stress induced by witnessing another person's plight. Therefore, despite the commonality among prosocial behaviors to increase another person's welfare broadly construed, we might actually be dealing with quite disparate motivations underlying these behaviors. Second, to understand the development of prosocial behavior in children, it seems critical to understand what psychological mechanisms are required for different prosocial behaviors. In particular, observed developmental trends towards greater prosocial behavior might not be explained by a change in the motivational component of prosocial behavior alone, but also by emerging social-cognitive abilities that enable older children to respond in prosocial ways in situations that are still beyond the scope of young children. The question thus arises, what affective, cognitive, and motivational abilities must be in place in order for children to act prosocially?

One mechanism underlying prosocial behavior that has gained a substantial following is empathy (Batson, 1991; Hoffman, 2000). Empathy and the associated process, sympathy, have been defined in a number of ways (Batson, 1998; Decety \& Jackson, 2006; Eisenberg \& Strayer, 1987; Feshbach, 1978; Hoffman, 1982; Preston \& de Waal, 2002; Wispé, 1986, 1987). Here, we will adopt a conceptualization offered by Nancy Eisenberg and colleagues (Eisenberg et al., 
1994; Eisenberg, Spinrad, \& Sadovsky, 2006; see also Hoffman, 1982; Hoffman, 2000) in which empathy is an affective response that stems from the apprehension or comprehension of another's emotional state and is similar to what the other person is feeling or would be expected to feel. Sympathy, on the other hand, is an affective response that stems from the apprehension or comprehension of another's emotional state but is not the same as the other's state, consisting instead of feelings of sorrow or concern for the other (Batson, 1987; Eisenberg, Shea, Carlo, \& Knight, 1991). Empathy and especially sympathy are thought to lead to prosocial behaviors such as helping and away from antisocial behaviors such as aggression (Batson, 1998; Batson et al., 1991; Eisenberg \& Miller, 1987; Hoffman, 1982, 2000; Miller \& Eisenberg, 1988).

The principal approach to investigating the ontogenetic development of this prosocial mechanism has been to assess how infants and young children respond to a victim's observable (visible or audible) emotional cues. In the typical paradigm, children are presented with a person (infant, mother, or stranger) displaying pain or distress, and children's empathic, sympathetic, and prosocial responses towards the victim (as evident in their facial, vocal, gestural, or physiological reactions) are assessed. This research suggests that soon after birth, infants automatically cry in response to other infants' cries (e.g., Sagi \& Hoffman, 1976; Simner, 1971). This global empathy is thought to be a precursor of empathy (Hoffman, 1982, 2000). Around 1214 months of age, infants show egocentric empathic distress, in which they respond to another's distress as if they themselves were in distress because they still lack a clear differentiation between self and other.

True or veridical empathy emerges in the second half of the second year, when children more fully differentiate between self and other and thereby understand that others are separate beings (as seen in their ability to recognize themselves in the mirror; Lewis, Sullivan, Stanger, \& 
Weiss, 1989). With this important cognitive development, toddlers realize that others have independent inner states, and now, when they see a person in distress after, say, bumping her knee or after her toy breaks, they show increasing empathic and sympathetic responses to others' visible distress as well as appropriate, other-directed comforting and prosocial behavior (e.g., Bischof-Köhler, 1991; Eisenberg \& Fabes, 1998; Zahn-Waxler \& Radke-Yarrow, 1982; ZahnWaxler, Radke-Yarrow, Wagner, \& Chapman, 1992; see Hoffman, 2000, for a detailed account of the developmental stages of empathic responding). Young children's empathy and sympathy have been found to relate positively with their prosocial behavior (Eisenberg \& Miller, 1987; Hoffman, 1982) and negatively with their antisocial and aggressive behavior (Miller \& Eisenberg, 1988), suggesting that empathic responding does indeed serve as a prosocial motive in early childhood.

Empathic responding to a victim's perceptible emotional cues can be aroused by multiple preverbal and automatic processes, including motor mimicry of the victim's emotional cues, emotional contagion, classical conditioning, and direct association of cues from the victim with one's own painful past experience (see Hoffman, 2000). These processes are argued to arouse an involuntary affective response and to require only a shallow level of cognitive processing (Hoffman, 2000). According to Hoffman, these modes are crucial for arousing empathy in early childhood but they continue to provide an involuntary route to empathy throughout life, especially in face-to-face situations in which the victim's distress is directly perceptible. Indeed, there is ample evidence to suggest that adults do empathize and sympathize with those in distress and that these empathic processes motivate their prosocial behavior (Batson et al., 1991; Batson, Duncan, Ackerman, Buckley, \& Birch, 1981; Decety \& Jackson, 2006; Singer \& Lamm, 2009). 
As all of this research shows, it is critical and extremely fruitful to investigate the emergence of children's empathic and prosocial responding towards a victim's emotional cues. Importantly, however, it has also been noted that empathic arousal is an especially important and reliable prosocial motivator because it is multi-determined (e.g., Hoffman, 2000). That is, empathic arousal can result in response to numerous types of distress cues by relying on several different modes of arousal, ranging from basic affective modes to cognitively sophisticated modes. This enables observers to respond empathically to whatever distress cues are available. Certainly, adults display a variety of empathic and prosocial behaviors, ranging from donating money to charities overseas to picking up a dropped object and handing it back to the owner, none of which require responding to overt emotional cues from the individuals in need. Our aim in this chapter is to present some recent work assessing young children's responses to such "situational cues," which we contrast with the overt emotional cues employed in much of the prior research on empathic and prosocial development. Furthermore, we will suggest that responding to such situational cues calls upon relatively sophisticated social-cognitive skills in addition to the affective and social-cognitive processes needed to respond to overt emotional cues.

We will focus on two types of situational cues. First, we will consider children's empathic and prosocial responses to distressing situations but in the absence of distress signals from the victim. We will present evidence that children in the second year can nevertheless sympathize with the victim and subsequently act prosocially towards her. Next, we will consider children's responses to concrete instrumental problems that may not require an apprehension of the other's emotional state but of a concrete instrumental problem in which a person cannot achieve her goal. We will present evidence that children in the second year of life respond by 
helping the person fulfill her goal. We will also consider the social-cognitive skills required to respond to each type of situational cues.

\section{Distressing situations in the absence of distress cues}

Although most prior work on the development of empathic responding has involved assessing children's responses to overt emotional cues, there are two lines of developmental research relevant to the question of how young children respond in the absence of overt distress signals. However, both lines of research are problematic. The first involves the use of picture and story assessments (Eisenberg-Berg \& Lennon, 1980; Feshbach \& Roe, 1968; Iannotti, 1985), in which the child is typically told brief stories while being shown pictures of hypothetical protagonists in emotion-eliciting situations (e.g., a child has lost her dog or a child is at a birthday party) but is given no information about the protagonists' feelings. After each story, the child is asked to indicate verbally or by pointing to pictures of facial expressions how she herself feels, under the assumption that the child has responded empathically if her reported emotion is similar to that of the protagonist. Eisenberg and Miller (1987) argue, however, that among other problems, these assessments create strong demand characteristics and thus tap children's inclination to provide the socially appropriate response rather than their empathic responses (see also Eisenberg \& Lennon, 1983; Eisenberg et al., 2006).

The second relevant line of research has examined children's affective perspectivetaking skills. Similar to the picture and story assessments, affective perspective-taking tasks typically involve the child being told stories about a character's situations and then being asked how the character feels. By 2.5 to 3 years of age, children are quite successful at these tasks, suggesting that young children have some understanding of others' affective states 
(Dunn \& Hughes, 1998; Harwood \& Farrar, 2006; Wellman, Phillips, \& Rodriguez, 2000; Wellman \& Woolley, 1990). However, these tasks do not test children's sympathy, i.e., it remains unclear whether, having comprehended the other's affective state, the child feels concern for the other and would act prosocially towards the other. Finally, a problem common to both lines of research is that the tasks used require relatively sophisticated cognitive abilities (e.g., the ability to understand hypothetical stories) and linguistic skills, which limits the age testable using these tasks.

We thus conducted a study to assess young children's empathic responses (specifically sympathy) towards a victim who shows no overt distress signals (Vaish, Carpenter, \& Tomasello, 2009). For this purpose, we adapted and modified a task developed by Hobson, Harris, García-Pérez, and Hobson (2009). In our study, 18-month-old and 2-yearold children first viewed an adult either harming another adult by destroying or taking away her possessions (harm condition) or else doing something similar that did not harm her (neutral condition). Importantly, the "victim" expressed no emotions in either condition, that is, she simply watched the other adult's harmful or harmless actions without displaying any emotional cues. Children viewed four such sympathy situations, during which we coded (1) the pattern of children's looks to the victim (e.g., the duration, latency, and number of looks, as well as the number of trials in which children looked to the victim), and (2) the quality of children's looks to the victim (whether each look was a concerned look or not; see Vaish et al., 2009, for details of the coding scheme).

Following these sympathy situations, we assessed children's prosocial behavior towards the victim in a subsequent prosocial situation. Recall that empathy and sympathy are thought to give rise to prosocial behavior, and infants' and children's empathic responding 
towards a victim displaying overt distress has previously been found to correlate with children's prosocial behavior towards the victim. Our reasoning was thus that if children are really sympathizing with a victim even in the absence of any distress cues (i.e., during our sympathy situations), then they should also subsequently show greater prosocial behavior towards the victim. In our prosocial situation, all children were given two balloons and the adult who had played the victim during the sympathy situations was given one balloon. After about a minute of individual play, the victim "accidentally" let go of her balloon (which floated to the ceiling), and was then vocally and facially (i.e., overtly) sad. Children's prosocial behavior was coded during the next 2 minutes (see Vaish et al., 2009, for details).

The results of this study showed clear differences in children's behavior across the two conditions. With regard to the pattern of looks, children in the harm condition looked to the victim more often, more quickly, and for longer than in the neutral condition. Moreover, children also showed greater sympathy for the victim in the harm condition than in the neutral condition. Specifically, more children showed concerned looks in the harm than in the neutral condition, and children showed concerned looks in a significantly greater number of the four harm situations than the four neutral situations.

Children's subsequent prosocial behavior towards the victim also differed significantly across conditions: Significantly more children acted prosocially towards (i.e., helped, comforted, or shared with) the victim if they had previously observed the victim in the harm rather than in the neutral condition. Furthermore, we found a significant correlation between children's sympathy and their subsequent prosocial behavior.

These findings are noteworthy for at least two reasons. First, they show that even very young children sympathize with victims and act prosocially towards them. This is in line with 
prior work, which showed that infants empathize and sympathize with those who overtly display distress and that this empathic responding mediates their prosocial behavior (Dunn, 1988; Eisenberg \& Fabes, 1998; Zahn-Waxler et al., 1992). More importantly, our findings show that by the middle of the second year of life, children can sympathize with a person in a distressing or harmful situation even if that person does not display overt distress signals, and that this sympathy also mediates children's subsequent prosocial behavior towards the victim.

What mechanisms are needed to respond sympathetically to such situational (rather than overt emotional) cues? Obviously, sympathy in the present study did not result directly from exposure to the victim's emotional cues (e.g., via mechanisms such as mimicking the emotional cues, emotional contagion, etc.), as such cues were not provided. It thus seems likely that sympathy in our study resulted at least partially from more sophisticated social-cognitive processes. One candidate social-cognitive process is affective perspective-taking, that is, drawing inferences about the other's affective state by putting oneself in the other's place and basing one's responses on those inferences (Eisenberg et al., 1991; Hoffman, 1984). In the absence of emotional cues, one way to make this inference is via simulation, which involves imagining oneself in another's situation (e.g., Decety \& Sommerville, 2003; Harris, 1995). An alternative but related possibility is that the observer can feel her way into the experience of and feel for the other person because she identifies with that person's attitudes (Hobson et al., 2009). In either case, one eventually takes the other's perspective and apprehends the other's affective state, which can activate affective responses such as sympathy and can thereby motivate prosocial behavior (Batson, Fultz, \& Schoenrade, 1987; Feshbach, 1978; Krebs \& Russell, 1981). Plausibly, then, in the present study, children apprehended the victim's state by taking her affective perspective, which motivated their sympathy and prosocial behavior. 
Depending on how familiar children were with situations like our sympathy situations, they might additionally have relied on their past experiences to infer the victim's affect. That is, if children had previously directly or vicariously experienced such situations on multiple occasions, perhaps they had formed scripts about people's responses to such situations and, in our study, were partially relying on these scripts to infer the victim's affect. On the other hand, if the situations were novel for children, then children likely engaged in perspective-taking (see Blair, 2005; Eisenberg et al., 1991; Karniol, 1982). Thus, perhaps some children in the harm condition (those familiar with such situations) relied less on affective perspective-taking and more on scripts than did children who were unfamiliar with such situations. However, even if the situations were to some degree familiar to children, it is highly unlikely that children had ever witnessed precisely the situations that they witnessed in our study (e.g., an adult tearing up another adult's drawing), and so although they might have had some scripts to rely on, they also had to engage in some affective perspective-taking.

In sum, our study demonstrates the flexible nature of empathy-related processes even in young children. As noted earlier, empathic arousal is thought to be a reliable prosocial motivator because it is multi-determined: The many modes of empathic arousal enable observers to respond empathically to whatever distress cues are available (Hoffman, 2000). Typically, victims are present and providing clear distress signals; in this case, any or several of the arousal mechanisms may be operating, ranging from the most basic and automatic forms of mimicry to the most advanced forms of cognitive reappraisal. However, when a victim is absent or is not providing distress signals for some reason, the cognitively advanced modes of empathic arousal still enable one to empathize, thus adding scope to one's empathic capability (Hoffman, 2000). Indeed, adults do empathize and sympathize with victims, both when they have direct access to 
the victims' distress cues and when they must engage more sophisticated cognitive processes, and these empathic processes do motivate their prosocial behavior (Batson et al., 1991; Batson et al., 1981; Decety \& Jackson, 2006; Ruby \& Decety, 2004; Singer \& Lamm, 2009). Our findings show that this multi-determined nature of empathy-related responses is already functional in early development. This suggests that empathy-related responses are indeed reliable proximate mechanisms underlying prosocial behavior even early in human ontogeny.

\section{Instrumental helping}

Beyond situations in which children respond and intervene to change the emotional states of others, young children also engage in acts of instrumental helping: A person is having trouble achieving a concrete goal (such as reaching for an out-of-reach object or being unable to open a door), and the child helps her achieve it (by moving the object closer or holding the door open for her). These instrumental helping behaviors are interesting motivationally, as they can provide insight into young children's willingness to act on behalf of others, especially in those cases where the child's actions are aimed at assisting with the other's goal-attainment rather than a benefit for oneself. These helping behaviors are also interesting cognitively, as they require the social-cognitive ability to represent other people's goals and intentions, rather than apprehending or resonating with the (presumed) emotional states of others. Thus, similar to the experiments on empathy and sympathy described above, instrumental helping critically depends upon children's ability to respond to situational cues and not facial expressions alone, with the difference that rather than inferring an emotional state, children have to be able to represent another person's action-goal.

It is a well-established phenomenon that by at least 12-18 months of age, children are able to represent other people's actions in terms of their underlying goals. Specifically, 
young children are able to differentiate between purposeful and accidental actions, highlighted for example in their tendency to imitate those aspects of an action that a person has done on purpose over those aspects the person has done only accidentally (Carpenter, Akhtar \& Tomasello, 1998). Moreover, they are able to infer what a person was trying but failing to do, even without actually witnessing the intended outcome (Meltzoff, 1998). Would young children utilize this social-cognitive capacity to read others' goals also to help others with their instrumental problems? A series of recent experiments show that from around 14 to 18 months of age, children begin to engage in instrumental helping, and do so in increasingly sophisticated ways over the second year of life.

In an initial study, we tested 18-month-old infants in a variety of situations in which an experimenter had trouble achieving his goal and the child had the opportunity to help (Warneken \& Tomasello, 2006). These situations presented the children with a variety of difficulties in discerning the person's goals. For instance, in one such situation, the person was hanging towels on a line, accidentally dropped a clothespin on the ground, and was unsuccessfully reaching for it. In another situation, the person was trying to put a stack of magazines into a cabinet, but he could not open the door because his hands were full and he bumped into the doors helplessly. Children helped in these situations by performing behaviors such as picking up the dropped object, opening the door, or completing the person's action after the failed attempt of stacking books. Importantly, children did not perform these actions in matched control conditions, in which the same basic situation and objects were presented, but there was no indication from the person that this constituted a problem for him (e.g., he dropped a clothespin on purpose). Therefore, these results indicated that children were able to infer the other person's goal in various contexts and knew how to 
intervene appropriately to help him achieve that goal. Another experiment showed that even 14-month-olds are willing and able to help instrumentally, although almost exclusively in situations in which helping consisted in handing over an out-of-reach object and not in more complex situations involving less salient goals and more complex forms of intervention (Warneken \& Tomasello, 2007).

The sophistication with which toddlers help instrumentally is also highlighted by the finding that they take into account what another person does or does not know. Specifically, in one helping test (Buttelmann, Carpenter, \& Tomasello, 2009), a protagonist put a toy into box A, after which the toy was moved to box B while the protagonist was either absent (ignorant condition) or present (knowledge condition). When the protagonist now unsuccessfully tried to open box A, 18-month-old children in the ignorant condition did not join him in trying to open box A, but instead opened box B in which the toy actually resided, indicating that they inferred that what the protagonist was actually trying to do was to get to the toy. Opening box A would not have helped him. However, children from the knowledge condition were more likely to open box A, presumably reasoning that the protagonist was not trying to get the toy which he knew had been moved to box B, and must thus have a new goal in mind for trying to open box A. This shows that these young children actually help others with their goals (which they infer based upon the other's state of knowledge) and do not blindly assist in completing any concrete action that another person (falsely) pursues.

Toddlers not only know when to help and how, but also whom. Specifically, 21month-old children will selectively help a person who was willing but unable to share a toy with them (because it rolled away) rather than a person who was unwilling to share a toy with them (i.e., she offered but then teasingly withdrew it) in a previous encounter (Dunfield 
$\&$ Kuhlmeier, 2010). That is, even though during the previous encounter the results of both individuals' actions were the same (the child did not receive a toy from either of them), they attended to the 'nice' person's intention to give them a toy and the 'mean' person's intention to not give them a toy. Therefore, children kept track of how each person had behaved towards them, and altered their own helping behavior accordingly. Moreover, 3-year-olds have also been shown to be selective in their helping behavior depending on how two individuals have behaved or intended to behave towards another person (rather than towards the children themselves). In this study (Vaish, Carpenter, \& Tomasello, in press), 3-year-olds selectively withdrew help from a person who had harmed another person or even intended (but failed) to harm another person. These studies thus demonstrate that young children rely on others' behaviors and intentions when selecting the beneficiaries of their helping acts.

Taken together, these experimental studies show that already early in ontogeny, children display impressive helping behaviors by intervening prosocially in a variety of ways. These findings highlight the sophisticated social-cognitive capacities that young children recruit to adequately represent other people's instrumental problems that go well beyond responding solely to others' overt distress cues.

\section{Conclusions and implications}

Empathic arousal is argued to be one of the most reliable and important motivators of prosocial behavior because it is multi-determined, that is, because it can be reached in response to many different types of cues, via various affective and cognitive routes (Hoffman, 2000). Yet,

until very recently, our understanding of the ontogenetic emergence of this motivator was limited to children's empathic and prosocial responses to overt, perceptible emotional cues, and thus to only a few and perhaps only the most basic affective mechanisms underlying empathic arousal. 
In this chapter, we aimed to go beyond this focus to present some recent work on young children's responses to situational cues and to explore the social-cognitive skills needed to respond to such cues.

Concerning the development of prosocial behavior, what is clear from the work we have presented is that already young children respond empathically and intervene prosocially towards others based upon situational cues. By at least 18 months of age, toddlers are able to be concerned for a person in a distressing situation but displaying no distress (Vaish et al., 2009). The social-cognitive skills required in order to respond in these ways include something akin to perspective-taking whereby, because the victim displays no distress, children must take her perspective in order to apprehend how she perceives the situation, which in turn enables them to sympathize with her. They cannot simply catch her emotion via emotional contagion, and they cannot simply identify how she feels by reading her overt emotional cues. Empathic responding in such a situation thus requires different if not greater social-cognitive processing than does empathic responding to overt distress cues.

Starting shortly after their first birthday, toddlers begin to engage in instrumental helping (Warneken \& Tomasello, 2006). Helping in these ways again requires different social-cognitive skills than empathic responding to overt distress cues: It requires appropriately inferring others' goals as well as the empathic or prosocial motivation to assist them in reaching their goals. In fact, already by 14 months, infants help others instrumentally in situations in which the goals are clear and in which intervention is simple, such as giving an object that is out of reach (Warneken $\&$ Tomasello, 2007). By at least 18 months of age, they can intervene fairly flexibly in different kinds of situations with more complex actions, such as when someone fails to open a door or uses the wrong means to achieve a goal (Warneken \& Tomasello, 2006). 
Thus, by 14 to 18 months of age, children display empathic responding to others' overt distress as well as in the absence of such distress but in the presence of other types of cues. Empathy and its related responses can thus motivate prosocial behavior in response to multiple cues, both emotional and situational, and via multiple routes, both affective and cognitive, even in early ontogeny, and are thus reliable prosocial motivators even in early ontogeny (see Hoffman, 2000).

These findings demonstrate the impressive flexible nature of empathic responding even in early childhood. Infants seem to come into the world equipped to automatically respond resonantly with others' emotions, as evident in their emotional contagion responses at birth (Sagi \& Hoffman, 1976; Simner, 1971). This way of responding to overt cues continues to function as a bottom-up and an at least partially involuntary route to empathy throughout life (Hoffman, 2000; Singer \& Lamm, 2009). However, with affective and cognitive development and experience, young children become better at apprehending others' inner states from increasingly varied types of cues, regulating their own inner states, keeping others' and their own inner states separate, and even inhibiting empathic and prosocial responding based on what they know about the beneficiary. By adulthood, empathic responding has become a highly flexible phenomenon that can be influenced by numerous top-down factors, including contextual (re)appraisal, the interpersonal relationship between empathizer and other, and the perspective adopted during observation of the other (Decety \& Jackson, 2006; Decety \& Lamm, 2006; Ruby \& Decety, 2004; Singer \& Lamm, 2009).

Furthermore, although we have focused here on the social-cognitive and affective contributors to empathic responding, to gain a fuller understanding, it is imperative to also consider the contributions of culture and experience. Indeed, research shows that the 
developmental context likely plays a critical and fascinating role. Although a comprehensive review of this work is beyond the scope of this chapter, it is worthwhile to consider a few examples to demonstrate the point. One recent study by Kärtner, Keller, and Chaudhary (2010), for instance, showed that although self-other differentiation has been thought for so long to be the critical requirement for true empathic responding (Hoffman, 1975) and has been shown to correlate with empathic responding in toddlers (e.g., Bischof-Köhler, 1991; Zahn-Waxler et al., 1992), in fact, this correlation only holds for children in cultural contexts in which parents emphasize individuality, autonomy, and self-reliance (in Kärtner et al.’s case, Germany). The correlation did not emerge in India, where parents emphasize social relationships and interpersonal responsiveness (obedience, prosocial behavior) over autonomy. Thus, Kärtner et al. (2010) argue that whereas empathic responding in Germany (and other cultures in which socialization goals concern autonomy) is grounded in perceiving the distinct subjective state that the other is in, that in India (and other cultures with relational socialization goals) has its origins in jointly experiencing the other person's distress. Perceiving the self as distinct from others may thus not be a universal requirement for the emergence of empathic responding.

There is also evidence of cultural and experiential influences on children's prosocial behavior (see Eisenberg, 1989, 1992). For instance, a recent study of 5-year-olds' spontaneous prosocial behavior revealed that German and Israeli children displayed more prosocial behavior towards a distressed adult as compared to Indonesian and Malaysian children (Trommsdorff, Friedlmeier, \& Mayer, 2007). The researchers propose that in cultures that promote face-saving values and respect for hierarchical relations (such as Indonesia and Malaysia), ignoring the mishap of another person (especially an authority figure) can be more valued than attempting to help and thereby risking that the other person lose face. Moreover, there is evidence that toddlers 
from abusive homes are much less likely to respond empathically or prosocially, and much more likely to respond with anger, fear, or physical attacks, to distressed peers than are toddlers from non-abusive homes (Main \& George, 1985). Such findings of variation across contexts are provocative because they highlight the ways in which culture and experience fundamentally shape empathic responding, and demonstrate vividly that empathic responding is not a unitary process but rather is open to a diverse set of influences. More generally, we propose that the relative automaticity of infants' initial affective response is transformed, with development, into a much more complex affective and cognitive response, one that is flexible and open to control, allowing us, in time, to experience forcefully and to respond effectively and in contextually appropriate ways to others' plight without being enslaved to those responses.

Finally, an important implication of the work we have presented is that both affect and cognition are important contributors to empathy-related responding. For instance, children in the Vaish et al. (2009) study were affectively involved in (and concerned about) a victim's plight; that, after all, was what we measured during the sympathy situations. However, since the victim provided no observable emotional cues, children likely had to rely on social-cognitive processes such as perspective-taking or relevant scripts in order to engage affectively with the victim. Moreover, they would also have needed to rely on the basic cognitive processes required for sympathizing and empathizing, such as self-other differentiation (although see Kärtner et al., 2010). This shows the integral link between affective and cognitive processes in producing empathic responding. Similarly, in Warneken and Tomasello's $(2006,2007)$ studies on instrumental helping, children had to rely on social-cognitive processes such as inferring unfulfilled goals but also needed a basic empathic or prosocial motivation to help the other fulfill his goals. Indeed, even empathic responding to overt, perceptible distress cues relies on both 
affective (e.g., catching and experiencing the other's emotion) and cognitive processes (e.g., selfother differentiation in order to keep the other's and one's own emotion separate). Thus, empathic responding in general is a complex, multi-faceted process. It is our hope that future work will continue to explore how flexible and sophisticated this mechanism underlying prosocial behavior truly is, especially in early childhood. 


\section{References}

Batson, C. D. (1987). Prosocial motivation: Is it ever truly altruistic? In L. Berkowitz (Ed.), Advances in experimental social psychology (Vol. 20, pp. 65-122). New York: Academic Press.

Batson, C. D. (1991). The altruism question: Toward a social-psychological answer. Hillsdale, NJ: Erlbaum.

Batson, C. D. (1998). Altruism and prosocial behavior. In D. T. Gilbert, S. T. Fiske \& G. Lindzey (Eds.), The handbook of social psychology (Vol. 2, pp. 282-316). Boston: McGraw Hill.

Batson, C. D., Batson, J. G., Slingsby, J. K., Harrell, K. L., Peekna, H. M., \& Todd, R. M. (1991). Empathic joy and the empathy-altruism hypothesis. Journal of Personality and Social Psychology, 61(3), 413-426.

Batson, C. D., Duncan, B. D., Ackerman, P., Buckley, T., \& Birch, K. (1981). Is empathic emotion a source of altruistic motivation? Journal of Personality and Social Psychology, 40(2), 290-302.

Batson, C. D., Fultz, J., \& Schoenrade, P. A. (1987). Adults' emotional reactions to the distress of others. In N. Eisenberg \& J. Strayer (Eds.), Empathy and its development (pp. 163185). Cambridge, UK: Cambridge University Press.

Bischof-Köhler, D. (1991). The development of empathy in infants. In M. E. Lamb \& H. Keller (Eds.), Infant development: Perspectives from German speaking countries (pp. 245-273). Hillsdale, NJ: Lawrence Erlbaum Associates.

Blair, R. J. R. (2005). Responding to the emotions of others: Dissociating forms of empathy through the study of typical and psychiatric populations. Consciousness and Cognition, $14,698-718$. 
Buttelmann, D., Carpenter, M., \& Tomasello, M. (2009). Eighteen-month-old infants show false belief understanding in an active helping paradigm. Cognition, 112, 337-342.

Decety, J., \& Jackson, P. L. (2006). A social-neuroscience perspective on empathy. Current Directions in Psychological Science, 15(2), 54-58.

Decety, J., \& Lamm, C. (2006). Human empathy through the lens of social neuroscience. The Scientific World Journal, 6, 1146-1163.

Decety, J., \& Sommerville, J. A. (2003). Shared representations between self and other: A social cognitive neuroscience view. Trends in Cognitive Sciences, 7, 527-533.

Dunn, J. (1988). The beginnings of social understanding. Cambridge, MA: Harvard University Press.

Dunn, J., \& Hughes, C. (1998). Young children's understanding of emotions within close relationships. Cognition and Emotion, 12, 171-190.

Eisenberg, N. (1989). The roots of prosocial behavior in children. Cambridge, UK: Cambridge University Press.

Eisenberg, N. (1992). The caring child. Cambridge, MA: Cambridge University Press.

Eisenberg, N., \& Fabes, R. A. (1998). Prosocial development. In N. Eisenberg (Ed.), Handbook of child psychology, Vol. 3: Social, emotional, and personality development (5th ed., pp. 701-778). New York: Wiley \& Sons.

Eisenberg, N., Fabes, R. A., Murphy, B., Karbon, M., Maszk, P., Smith, M., et al. (1994). The relations of emotionality and regulation to dispositional and situational empathy-related responding. Journal of Personality and Social Psychology, 66, 776-797.

Eisenberg, N., \& Lennon, R. (1983). Sex differences in empathy and related capacities. Psychological Bulletin, 94, 100-131. 
Eisenberg, N., \& Miller, P. A. (1987). The relation of empathy to prosocial and related behaviors. Psychological Bulletin, 101, 91-119.

Eisenberg, N., Shea, C. L., Carlo, G., \& Knight, G. P. (1991). Empathy-related responding and cognition: A "chicken and the egg" dilemma. In W. Kurtines \& J. Gewirtz (Eds.), Handbook of moral behavior and development, Vol. 2: Research (pp. 63-88). Hillsdale, NJ: Lawrence Erlbaum Associates.

Eisenberg, N., Spinrad, T. L., \& Sadovsky, A. (2006). Empathy-related responding in children. In M. Killen \& J. G. Smetana (Eds.), Handbook of moral development (pp. 517-549). Mahwah: Lawrence Erlbaum Associates.

Eisenberg, N., \& Strayer, J. (1987). Critical issues in the study of empathy. In N. Eisenberg \& J. Strayer (Eds.), Empathy and its development (pp. 3-13). Cambridge, UK: Cambridge University Press.

Eisenberg-Berg, N., \& Lennon, R. (1980). Altruism and the assessment of empathy in the preschool years. Child Development, 51, 552-557.

Feshbach, N. D. (1978). Studies of empathic behavior in children. In B. A. Maher (Ed.), Progress in experimental personality research (Vol. 8, pp. 1-47). New York: Academic Press.

Feshbach, N. D., \& Roe, K. (1968). Empathy in six- and seven-year-olds. Child Development, $39,133-145$.

Harris, P. L. (1995). From simulation to folk psychology: The case for development. In M. Davies \& T. Stone (Eds.), Folk psychology: The theory of mind debate (pp. 207-231). Oxford: Blackwell. 
Harwood, M. D., \& Farrar, M. J. (2006). Conflicting emotions: The connection between affective perspective taking and theory of mind. British Journal of Developmental Psychology, 24, 401-418.

Hay, D. F., \& Cook, K. V. (2007). The transformation of prosocial behavior from infancy to childhood. In C. A. Brownell \& C. B. Kopp (Eds.), Socioemotional Development in the Toddler Years (pp. 100-131). New York: The Guilford Press.

Hobson, J. A., Harris, R., García-Pérez, R., \& Hobson, P. (2009). Anticipatory concern: A study in autism. Developmental Science, 12(2), 249-263.

Hoffman, M. L. (1975). Developmental synthesis of affect and cognition and its implications for altruistic motivation. Developmental Psychology, 11(5), 607-622.

Hoffman, M. L. (1982). Development of prosocial motivation: Empathy and guilt. In N. Eisenberg (Ed.), The development of prosocial behavior (pp. 281-338). New York: Academic Press.

Hoffman, M. L. (1984). Interaction of affect and cognition in empathy. In C. E. Izard, J. Kagan \& R. B. Zajonc (Eds.), Emotion, cognition, and behavior (pp. 103-131). Cambridge, UK: Cambridge University Press.

Hoffman, M. L. (2000). Empathy and moral development: Implications for caring and justice. Cambridge, UK: Cambridge University Press.

Iannotti, R. J. (1985). Naturalistic and structured assessments of prosocial behavior in preschool children: The influence of empathy and perspective taking. Developmental Psychology, 21(1), 46-55.

Karniol, R. (1982). Settings, scripts, and self-schemata: A cognitive analysis of the development of prosocial behavior. In N. Eisenberg (Ed.), The development of prosocial behavior (pp. 251-278). New York: Academic Press. 
Kärtner, J., Keller, H., \& Chaudhary, N. (2010). Cognitive and social influences on early prosocial behavior in two sociocultural contexts. Developmental Psychology, 46(4), 905914.

Krebs, D. L., \& Russell, C. (1981). Role-taking and altruism: When you put yourself in the shoes of another, will they take you to their owner's aid? In J. P. Rushton \& R. M. Sorrentino (Eds.), Altruism and helping behavior (pp. 137-165). Hillsdale, NJ: Erlbaum.

Lewis, M., Sullivan, M. W., Stanger, C., \& Weiss, M. (1989). Self development and selfconscious emotions. Child Development, 60(1), 146-156.

Main, M., \& George, C. (1985). Responses of young abused and disadvantaged toddlers to distress in agemates. Developmental Psychology, 21(3), 407-412.

Miller, P. A., \& Eisenberg, N. (1988). The relation of empathy to aggressive and externalizing/antisocial behavior. Psychological Bulletin, 103, 324-344.

Preston, S. D., \& de Waal, F. B. M. (2002). Empathy: Its ultimate and proximate bases. Behavioral and Brain Sciences, 25, 1-72.

Ruby, P., \& Decety, J. (2004). How would you feel versus how do you think she would feel? A neuroimaging study of perspective-taking with social emotions. Journal of Cognitive Neuroscience, 16(6), 988-999.

Sagi, A., \& Hoffman, M. L. (1976). Empathic distress in newborns. Developmental Psychology, $12,175-176$.

Simner, M. L. (1971). Newborns' response to the cry of another infant. Developmental Psychology, 5, 136-150.

Singer, T., \& Lamm, C. (2009). The social neuroscience of empathy. Annals of the New York Academy of Sciences, 1156, 81-96. 
Trommsdorff, G., Friedlmeier, W., \& Mayer, B. (2007). Sympathy, distress, and prosocial behavior of preschool children in four cultures. International Journal of Behavioral Development, 31(3), 284-293.

Vaish, A., Carpenter, M., \& Tomasello, M. (2009). Sympathy through affective perspectivetaking and its relation to prosocial behavior in toddlers. Developmental Psychology, 45(2), 534-543.

Vaish, A., Carpenter, M., \& Tomasello, M. (in press). Young children selectively avoid helping people with harmful intentions. Child Development.

Warneken, F., \& Tomasello, M. (2006). Altruistic helping in human infants and young chimpanzees. Science, 311, 1301-1303.

Warneken, F., \& Tomasello, M. (2007). Helping and cooperation at 14 months of age. Infancy, 11(3), 271-294.

Warneken, F., \& Tomasello, M. (2009). The roots of human altruism. British Journal of Psychology, 100(3), 455-471.

Wellman, H. M., Phillips, A. T., \& Rodriguez, T. (2000). Young children's understanding of perception, desire, and emotion. Child Development, 71, 895-912.

Wellman, H. M., \& Woolley, J. D. (1990). From simple desires to ordinary beliefs: The early development of everyday psychology. Cognition, 35, 245-275.

Wispé, L. (1986). The distinction between sympathy and empathy: To call forth a concept, a word is needed. Journal of Personality and Social Psychology, 50, 314-321.

Wispé, L. (1987). History of the concept of empathy. In N. Eisenberg \& J. Strayer (Eds.), Empathy and its development (pp. 17-37). New York: Cambridge University Press. 
Zahn-Waxler, C., \& Radke-Yarrow, M. (1982). The development of altruism: Alternative research strategies. In N. Eisenberg (Ed.), The development of prosocial behavior (pp. 109-137). New York: Academic Press.

Zahn-Waxler, C., Radke-Yarrow, M., Wagner, E., \& Chapman, M. (1992). Development of concern for others. Developmental Psychology, 28(1), 126-136. 